$\Delta$ Palabras clave/ Renacimiento, urbanismo città idéale, humanistas.

$\Delta$ Keywords/ Renaissance, urban planning, città ideale, humanists.

$\Delta$ Recepción/ 29 julio 2015

$\Delta$ Aceptación/ 9 septiembre 2015

\section{La renovación urbana en las ciudades ideales italianas siglos XV y XVI}

\section{Urban renovation in Italian ideal cities during the fifteenth and seventeenth centuries.}

\author{
David Hidalgo García \\ Graduado en ciencias y tecnologías de la \\ edificación, Universidad de Sevilla, España \\ Doctor por la Universidad de Alicante, España. \\ Profesor docente e investigador del \\ Departamento de expresión gráfica \\ arquitectónica y en la ingeniería, Universidad \\ de Granada, España. \\ dhidalgo@ugr.es
}

\section{Juan Manuel Santiago Zaragoza}

Arquitecto Técnico y licenciado en empresariales, Universidad de Granada, España. Doctor por la Universidad de Granada, España. Profesor docente e investigador del Departamento de expresión gráfica arquitectónica y en la ingeniería, Universidad de Granada, España.

santi@ugr.es

\section{Julián Arco Díaz}

Arquitecto Técnico, Universidad de Granada, España. Doctorando de la Universidad de Almería, España

Profesor docente e investigador del Departamento de expresión gráfica arquitectónica y en la ingeniería, Universidad de Granada, España.

juliannnaugr.es

RESUMEN/ Durante el Renacimiento se modificaron los estilos de vida, las costumbres sociales y el pensamiento de la sociedad. Esto supuso una modificación respecto a las actitudes y comportamientos en relación a la organización de las ciudades que, principalmente dentro del ámbito italiano de los siglos XV y XVI, originó el nacimiento de una serie de propuestas encaminadas al diseño y desarrollo de una ciudad perfecta, conocida con el nombre de città idéal. Estas fueron creadas y estudiadas por un grupo de arquitectos humanistas que defendieron la teoría de que las mismas constituían un organismo urbano, planificable en todas sus partes y que, por tanto, podían cumplir con los objetivos de defensa militar y corrección de las deficiencias higiénicas que arrastraban las ciudades hasta ese momento. La finalidad del presente artículo es la realización de un análisis crítico de estas propuestas con el objetivo de obtener parámetros comunes y diferenciadores. ABSTRACT/ Renaissance meant a change in lifestyles, social customs and society's way of thinking. This entailed a shift in the attitudes and behavior towards the layout of cities, which -mainly during the fifteenth and sixteenth centuries in Italy- gave birth of several proposals for the design and development of a perfect city, known by the name of the Ideal City (Italian città ideale). These proposals were developed and studied by a group of humanist architects who defended the theory that such cities made up an urban body subject to planning in all its parts, and which could therefore meet military defense goals and improve poor sanitation conditions, borne by cities for a long time. The purpose of this article is to carry out a critical analysis of these proposals in order to achieve common and differentiating parameters.

\section{INTRODUCCIÓN. En la cultura}

\section{renacentista, la proyección de la ciudad} constituyó un elemento primordial de gran importancia. Su desarrollo y estudio adquirió una singular relevancia dentro de la sociedad ya que, desde mediados del siglo XIII, se inició en las ciudades un proceso de decoro urbano' y de regulación de los espacios, tanto públicos como privados. Las intervenciones se desarrollaron sobre elementos individuales, aunque en algunos casos, afectaban a la globalidad de la urbe (Franchetti 1985).
El proceso continuó mediante la elaboración de una serie de normas y reglamentaciones ${ }^{2}$ que controlaban las actividades y edificaciones ${ }^{3}$ de la ciudad y finalizó en los primeros decenios del siglo XV con la aspiración de llegar a una nueva concepción de urbe, conocida con el nombre de città idéale $e^{4}$

Según lo indicado por el autor Vittorio Franchetti (1985), las líneas según las cuales se desarrolló el proceso de renovación de las ciudades del Renacimiento fueron cuatro: mejora del decoro urbano de las urbes, búsqueda de nuevos tipos de construcción ampliación de las ciudades y, por último, la búsqueda teórica de la completa renovación urbana, es decir, la creación de la ciudad ideal. El autor Evelio Moreno va mucho más allá y afirma: "La città idéale de los renacentistas italianos es la alternativa al paraíso. [. . . ] Este es sustituido por una città como comunidad de vida y pensamiento" (Moreno 1991).

${ }^{2}$ El objetivo fundamental de la normativa y reglamentación aprobada era el decoro de las ciudades. El decoro se entendía no solo como la estética de las edificaciones sino también en relación a la moralidad y las costumbres, tales como: higiene en la edificación, desplazamiento de las actividades ruidosas y malolientes a las zonas exteriores, equipamientos urbanos para favorecer el almacenamiento 
En los inicios, la città idéa/e $e^{5}$ ue pensada como un elemento global de bienestar para los ciudadanos y no como un conjunto de calles y viviendas dotadas de unas infraestructuras desconocidas hasta ese momento. Su estudio y desarrollo tuvo una doble vertiente: por un lado una línea en la que la ciudad se podía asimilar a un organismo, conocida con el nombre antropomórfica (imagen 1), cuyos máximos exponentes fueron los arquitectos ${ }^{6}$

Francesco di Giorgio Martini, Baldassarre Peruzzi y Antonio Averlino 'Filarete'. En la línea opuesta, se encuentran arquitectos tales como León Battista Alberti, Francesco de Marchi o Vincenzo Scamozzi, para los que la ciudad no debía tener relación con el cuerpo humano y su función no dejó de ser otra que la de albergar y satisfacer las necesidades de las personas. En algunos casos no solo necesidades físicas sino también psíquicas o de bienestar. El estudio de las ciudades ideales constituyó una de las mayores y mejores contribuciones urbanísticas realizadas a lo largo de la historia. Estas quedaron reflejadas y analizadas, fundamentalmente, en los distintos tratados realizados durante el Renacimiento y dentro del territorio italiano (Morris 2007).

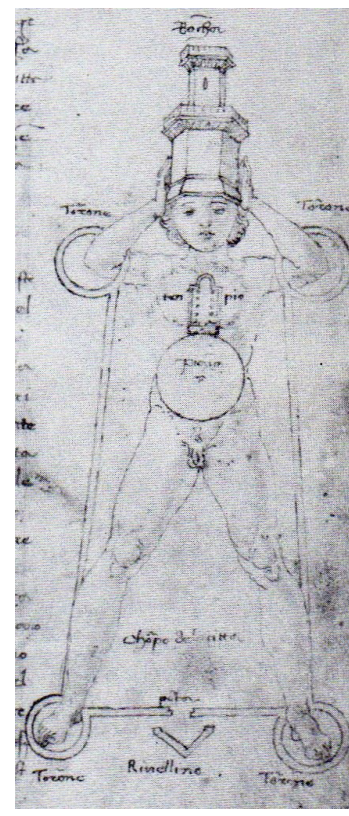

Imagen 1. Relación entre la ciudad y el cuerpo humano (fuente: Giorgio 1967).

\section{LAS CITTÀS IDÉALES ITALIANAS SIGLOS XV Y XVI.}

Se puede indicar que el nacimiento de estas propuestas teóricas ${ }^{7}$ de renovación urbana surgieron como consecuencia de:

$\left.\mathbf{1}^{\circ}\right)$ Defensa Militar: Durante la Edad Media los precarios sistemas defensivos de las ciudades eran suficientes para resistir el asalto producido mediante las armas de fuego. Tras la invención del cañón se exigió que los sistemas defensivos sufrieran un gran cambio para poder resistir los asaltos (Croix 1960). Por este motivo, se diseñaron ciudades resistentes al asedio externo ${ }^{8}$, tanto a nivel de sistemas defensivos y de distribución de la morfología urbana, como a nivel de capacidad de autosuficiencia durante un largo periodo en caso de asedio. $\left.2^{\circ}\right)$ Corrección de deficiencias urbanas: Las nuevas propuestas tienden a contar con espacios libres, acondicionamiento de calles e instalaciones de salubridad. Dichos planes llegaron a unos niveles excepcionales con ciertos arquitectos. Por ejemplo, los estudios de las condiciones de soleamiento de las calles y viviendas realizados por Pietro Cataneo y Leonardo da Vinci, o en los estudios de ventilación de viviendas realizados por León Battista.
En los tratados italianos de los siglos XV y XVI estudiados (tabla 1), se observa una evolución con respecto a la documentación teórica y práctica de sus cittàs idéales. Esta evolución, en cierta medida, es lógica y viene motivada por la cronología temporal en la que se escribieron. Las fechas de finalización de cada tratado son correlativas, por lo que cada autor tuvo la oportunidad de estudiar y consultar el tratado de sus antecesores. A esto, hay que añadir que Baldassarre Peruzzi fue aprendiz de Francesco di Giorgio y maestro de Pietro Cataneo. Esta circunstancia se aprecia claramente en los tratados de los dos primeros, los cuales presentan unas similitudes importantes en sus estudios de ciudades

Pero también existen diferencias en las propuestas de estos autores que fueron maestros y aprendices. Por ejemplo, Pietro Cataneo que fue el último aprendiz de la secuencia, no utilizó en sus estudios la tipología de ciudad radio concéntrica sino una cuadrícula ortogonal con manzanas irregulares junto a las murallas. Este elemento fue usado posteriormente por Vincenzo Scamozzi en sus estudios de ciudad ideal. En las propuestas de ambos
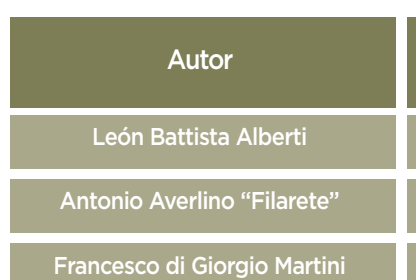

Francesco di Giorgio Martin

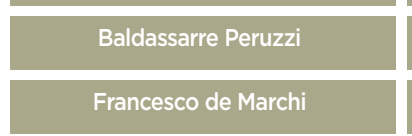

Giovanni Battistta Bellucci
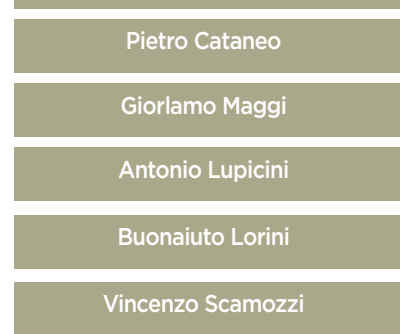

Tabla 1. Relación de arquitectos del Renacimiento con fecha de vida y de publicación de Trata

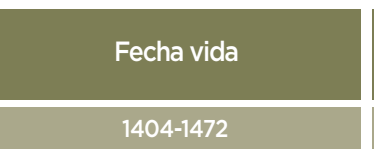

\begin{tabular}{|c|}
\hline $1402-1472$ \\
\hline $1429-1502$ \\
\hline
\end{tabular}

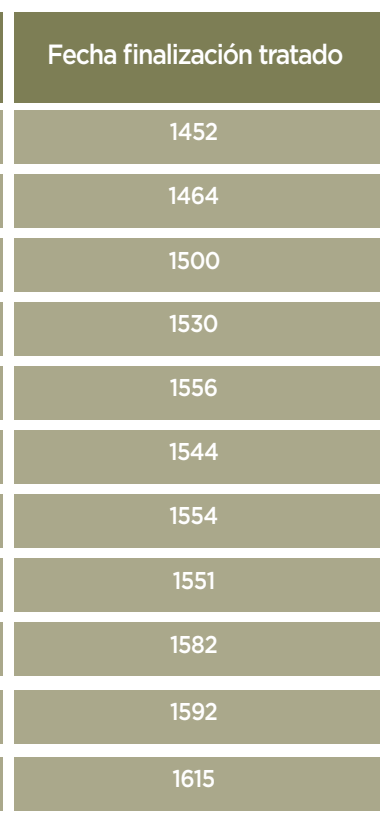

\begin{tabular}{|l|l|}
\hline 1452 \\
\hline 1464 \\
\hline 1530 \\
\hline 1556 \\
\hline 1544 \\
\hline 1554 \\
\hline 1551 \\
\hline 1582 \\
\hline 1592 \\
\hline 1615
\end{tabular}

$1504-1576$

$1506-1554$

\begin{tabular}{|c|}
\hline $1510-1569$ \\
\hline $1523-1572$ \\
\hline
\end{tabular}

$1530-1607$

\begin{tabular}{|l|l|}
$1530-1611$ \\
\hline $1548-1616$
\end{tabular}

Fecha finalización tratado

\section{?}

${ }^{5}$ En relación a la ciudad ideal, son interesantes los comentarios del autor Puppi (1973). ${ }^{6}$ No obstante, la primera idea en relación a la proporción de las ciudades a partir del cuerpo humano fue tenida en cuenta por Luca Pacioli en su publicación titulada Divina proportione escrita entre los

${ }^{7}$ El utilizar el término 'teórico' es porque no existe constancia de la construcción de ninguna de estas propuestas de città idéale durante el Renacimiento.

${ }^{8}$ Para saber más en relación a la influencia de las armas de fuego en el diseño de las ciudades, consultar: Vagnetti (1974). 


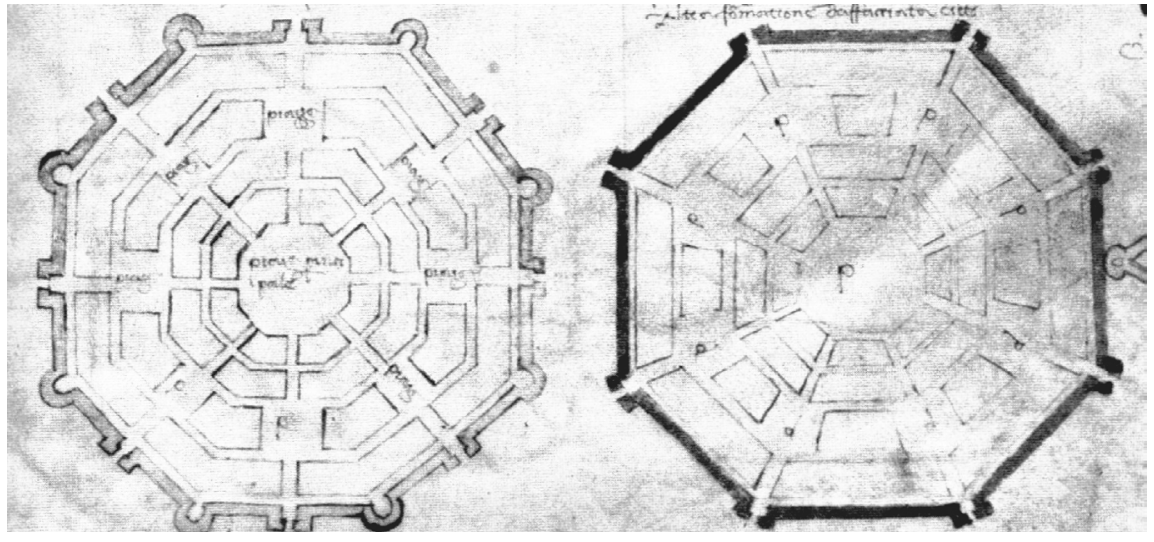

Imagen 2. Propuestas de ciudades en llano Francesco di Giorgio (fuente: Giorgio 1967)
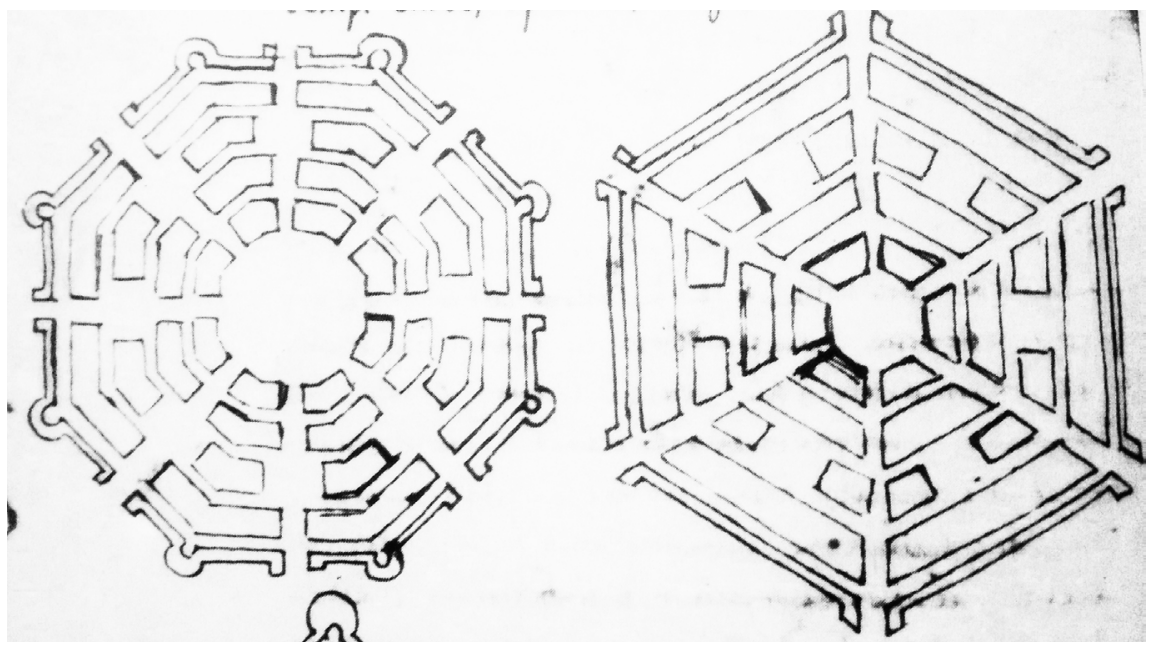

Imagen 3. Propuestas de ciudades en llano, de Baldassarre Peruzzi (fuente: Peruzzi 1530).
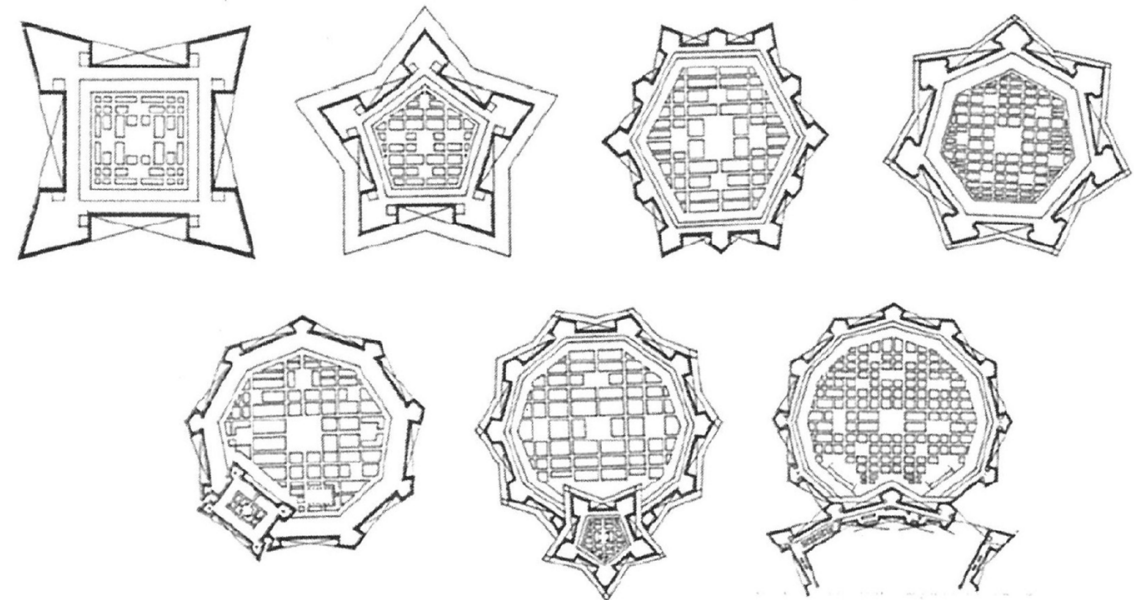

Imagen 4. Propuestas de ciudades en /lano (fuente: Cataneo 1554).

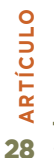

Aunque el arquitecto romano Vitruvio, en su De Architectura escrito en el año 80 a.C. contempló una distribución radial. autores, las manzanas próximas a la muralla presentan formas irregulares en contraposición al resto de manzanas en retícula. El objetivo de estas era aprovechar el espacio disponible entre la manzana anexa y la muralla. En imagen 2, imagen 3 e imagen 4 se puede observar las similitudes entre Francesco di Giorgio y Baldassarre Peruzzi y las diferencias entre estos y Piero Cataneo, ya indicadas

De todos los tratados indicados en la tabla 1, el primero por orden cronológico es el del arquitecto León Battista Alberti. Este carece de indicaciones prácticas que complementen las descripciones teóricas que traslada en su tratado. Por este motivo, el autor Morris lo nombra como "el primer teórico del Renacimiento" (Morris 2007). Dicho elemento se corrige posteriormente en los tratados de Antonio Averlino

'Filarete', , Francesco di Giorgio y Francesco de Marchi, que contienen una importante documentación gráfica (Muratore 1980)

Destacan las ilustraciones de Marchi, consideradas por algunos autores como las mejores representaciones de ciudades del Renacimiento (Guidoni 1985)

A partir de aquí, se desarrollaron otros tratados con el objetivo de cumplir con las premisas impuestas para las ciudades ideales. Para ello, todos los autores indicados, salvo Cataneo y Scamozzi, plantearon ciudades con un sistema de distribución radial. El sistema se inició a mediados del siglo XV con la propuesta básica de Antonio Averlino 'Filarete' y resultó ser una herramienta habitual en los tratadistas del Renacimiento (Croix 1960). Su máximo desarrollo fue llevado a cabo por Francesco di Giorgio en el año 1500 quién, además, propuso una alternativa al plan radial mediante ciudades con desarrollo en retícula. Esta otra propuesta fue asumida posteriormente por Marchi en el año 1556, aunque al igual que Di Giorgio, en líneas generales prefirió ciudades radioconcéntricas. No es hasta finales del siglo $\mathrm{XVI}$ cuando el trazado reticular (imagen 4) desbancó al trazado radial mediante los tratados de los autores Pietro Cataneo ${ }^{10}$ y Vicenzo Scamozzi. 
Por lo tanto, las propuestas predominantes de ciudades ideales del Renacimiento italiano se dividen en dos grandes grupos: 1) Ciudades radio concéntricas. Todas ellas, con mayores o menores similitudes, disponen de murallas de protección, morfología urbana con distribución interior radio concéntrica y en su punto central se ubica la plaza de armas". Las propuestas más desarrolladas planteaban la necesidad de espacios públicos secundarios para albergar mercados, otros edificios principales y zonas de esparcimiento. Dentro de este tipo de ciudades es necesario hacer mención a un tema importante y relacionado con el objetivo de defensa militar por el cual nacieron. En caso de asedio, las tropas debían trasladarse rápidamente desde la Plaza de Armas (ubicada en el centro de la ciudad) a los bastiones de las murallas. Para cumplir con este objetivo, los autores descritos planteaban ciudades con calles que unían estos dos elementos arquitectónicos, tal y como se puede observar en la imagen 5. De esta forma, al contar con conexión directa, el traslado entre ambos lugares se realizaba en el menor tiempo posible.

Entre todas las propuestas se pueden encontrar otras que no cumplen con esa premisa (ver imagen 6). En ellas los bastiones no tienen conexión directa con la Plaza de Armas.
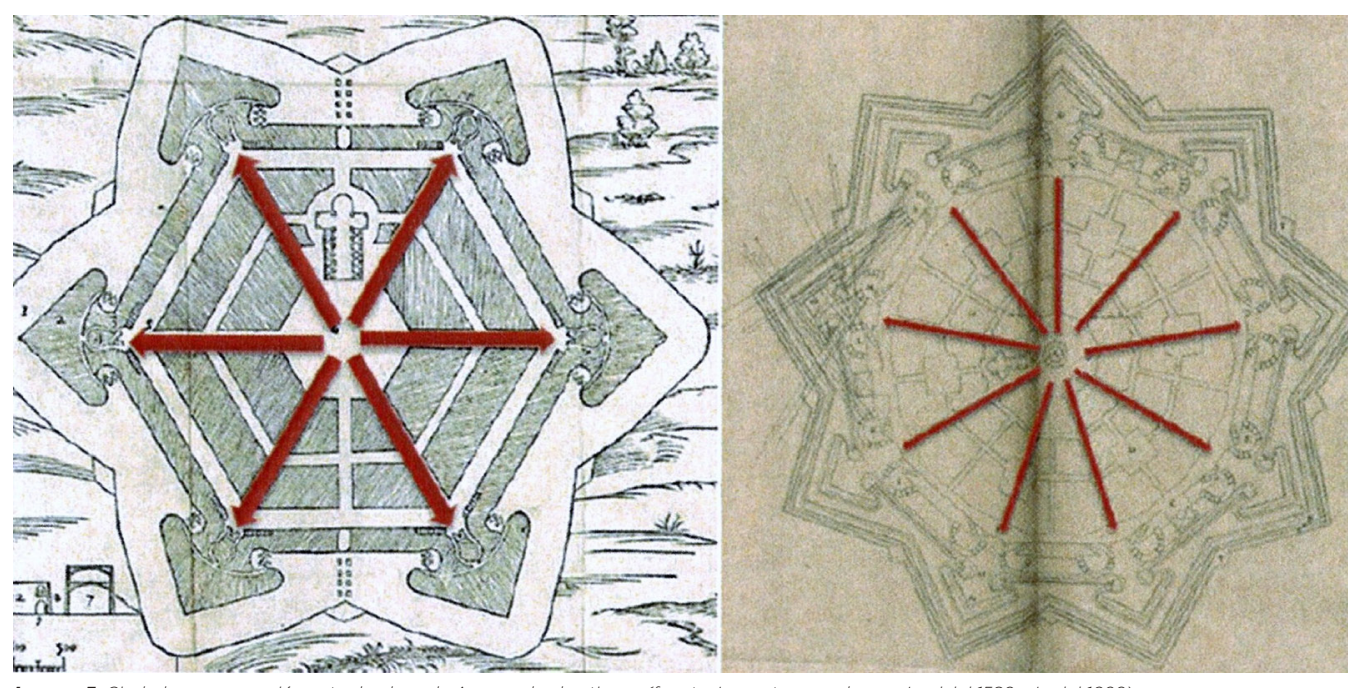

Imagen 5. Ciudades con conexión entre la plaza de Armas y los bastiones (fuente: Los autores en base a Lupicini 1582 y Lorini 1609).
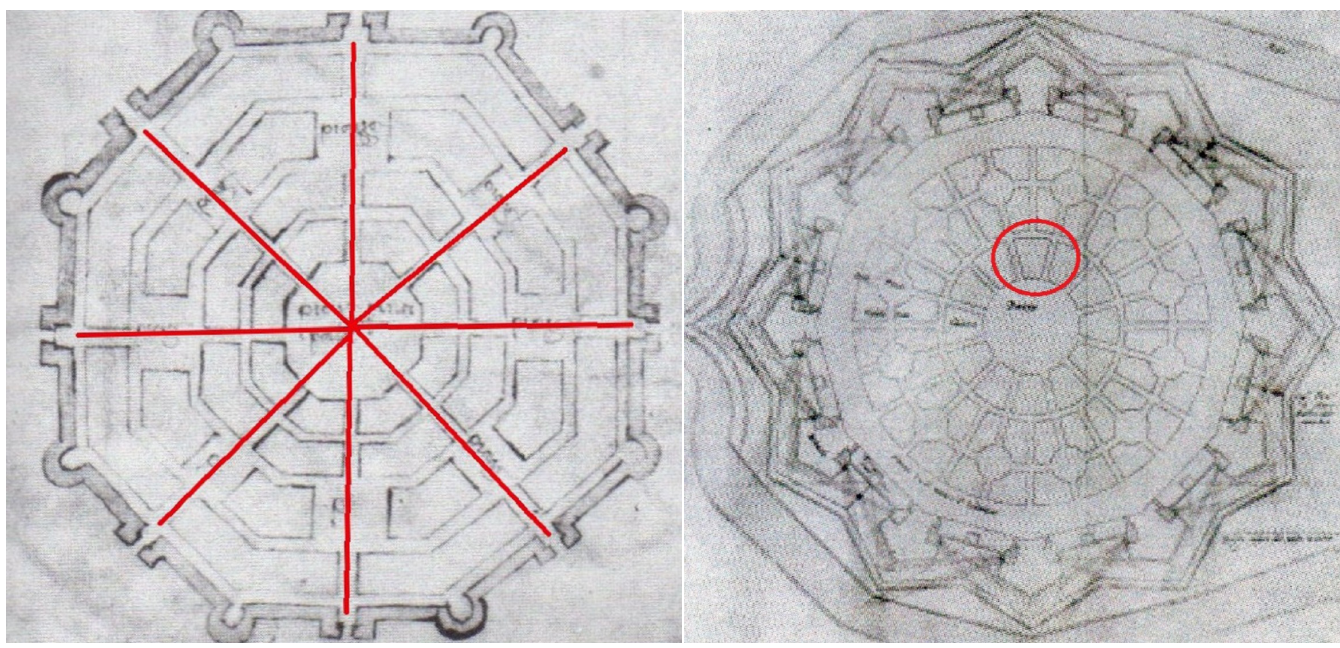

Imagen 6. Ciudades sin conexión entre la plaza de Armas y los bastiones (fuente: Los autores en base a Giorgio 1482 y Marchi 1556).

En la propuesta de Francesco di Giorgio, que puede observarse en la imagen 6, la Plaza de Armas conecta con las puertas de la ciudad, no disponiendo los bastiones de enlace directo con el punto central de la urbe. Lo mismo ocurre con la propuesta de Francesco de Marchi que aunque plantea calles que parten de los bastiones, estas son interrumpidas por manzanas de viviendas antes de llegar al centro. La teoría en relación a estas otras propuestas es indicada por algunos autores (Croix 1960) y se relaciona con la idea de conectar el centro de la ciudad con las puertas de acceso. Dicha circunstancia se desaconsejaba, ya que en caso de entrada de las tropas enemigas, se facilitaba su llegada al centro de la ciudad y, por tanto, a los edificios de gobierno ${ }^{12}$. No obstante, estas propuestas son las menos planteadas, posiblemente por contar con ese inconveniente. 
3. CONCLUSIONES. A finales del siglo XIV, y como consecuencia del aumento de población y de concentración en las ciudades, empezaron a aparecer una serie de reglamentaciones y normas que intentaban corregir las deficiencias de salubridad que presentaban las urbes. El Renacimiento italiano fue un cúmulo de circunstancias que rompió con la tendencia que se venía desarrollando en épocas anteriores. Implicó el renacer de la civilización y la salida del periodo de oscuridad en el que se encontraba la humanidad. Este renacer también se aplicó a las ciudades y a su desarrollo, motivado fundamentalmente por dos elementos importantes: el surgimiento de un grupo de arquitectos humanistas con otras teorías respecto a las ciudades y el desarrollo de nuevas técnicas constructivas que permitían la construcción de elementos inimaginables hasta ese momento, tales como los excelentes sistemas defensivos capaces de resistir los ataques de armas de fuego o las cúpulas de los Duomos de Florencia y Milán, consideradas por algunos autores como las obras más importantes del Quattrocento italiano.
Las ciudades utópicas del Renacimiento se desarrollaron con los objetivos de defensa militar y corrección de las condiciones de insalubridad que se venían desarrollando en las ciudades de la Edad Media. De esta forma, empezaron a aparecer publicados una serie de tratados arquitectónicos y militares que cambiaron el curso de las ciudades, transformándolas de urbes medievales con calles estrechas, tortuosas y sin instalaciones de salubridad en ciudades con espacios abiertos, de calles rectas y donde se tenían en cuenta las condiciones de soleamiento, ventilación y salubridad de las viviendas.

Básicamente, consistían en ciudades amuralladas con distribución radio concéntrica o reticular con amplios espacios libres en su interior, instalaciones de salubridad y reubicación de actividades insalubres en zonas alejadas de las residenciales. La distribución radio concéntrica tuvo cabida hasta mediados del siglo XV, cuando fue sustituida por la distribución reticular. En algunos casos se indicaban las mejores condiciones de orientación de las viviendas para mejorar su salubridad, ventilación y soleamiento.
Contaban con calles con pendientes para favorecer la evacuación de las aguas de lluvia y con canales o ríos para el abastecimiento y la evacuación. Se estudiaba conscientemente su ubicación para garantizar climas confortables y excelentes zonas anexas de cultivo. De esta forma, se obtuvieron cuatro tipos principales de ciudades: en llano, en monte, marítimas y atravesadas por ríos.

Eran ciudades diseñadas, estudiadas y creadas para y por los ciudadanos y con el objetivo fundamental de cubrir sus necesidades. Posiblemente la del Renacimiento ha sido la línea de ciudades utópicas más perfecta y completa que se ha desarrollado a lo largo de la historia, solo comparable, y en menor medida, con la de los utopistas del siglo XIX.

\section{REFERENCIAS}

Arévalo. F. 2000. La representación de la ciudad en el Renacimiento: levantamiento urbano y territorial (tesis doctoral Escuela Técnica Superior de Arquitectura). Sevilla: Universidad de Sevilla.

Calatrava. J. y Nerdinger, W. 2010. Arquitectura escrita. Madrid: Circulo de bellas artes, 105-111. Cataneo. P. 1554. / quattro libri del/' architettura. (Consultado el 28 de Junio de 2015). Disponible en: http://fondosdigitales.us.es/fondos/libros/1708/17/i-quattro-primi-libri-di-architettura-di-pietro-cataneo-senese/ Croix, H., 1960. "Military architecture and the radial city plan in sixteenth century Italy." The art Bulletin. XLII. 42 (4), 263-290,

Franchetti, V., 1985. Historia del urbanismo: Siglos XIV y XV. Instituto de Estudios de Administración Local. Madrid.

Fuente. M. J., 1991. "La ciudad de Dios. El factor religioso en la construcción de la ciudad medieval." Historia, 16. Año XVI, 186, 51-60

Giorgio, F., 1967. Tratatti di architettura, ingegneria e arte militare (Vol. I y II). Milán: Ediciones C. Maltese. Guidoni, E., 1985. Historia del urbanismo. El siglo XVI. Madrid: Instituto de estudios de administración local. Lorini, B., 1609. Delle fortificatione libri cinque. Venecia.
Lotz, W., 1976. Studies in Italian Renaissance architecture. Massachusetts: MIT Press. Lupicini, A., 1582. Architettura militare con altri avvertimenti apparteneti alla querra. Florencia. Marchi, F., 1556. Della architettura militare. Moreno, E., 1991. Las ciudades ideales de/ siglo XVI. Barcelona: Edictorial Sendai. Morris, A., 2007. Historia de la forma urbana. Barcelona: Editorial Gustavo Gili. Muratore, G., 1980. La ciudad renacentista: tipos y modelos a través de los tratados. Madrid: Instituto de estudios de administración local.

Pedretti, C., 1988. Leonardo architetto. Milán: Ediciones Electa. Peruzzi, B., 1530. Trattato de architettura militare. Edición consultada en la Biblioteca del Hospital Real de Granada. Universidad de Granada.

Puppi, L., 1973. "La citta idéale nella cultura architettonica del Rinascimento europeo." En Atti del XXI Congresso Internazionale di storia dell'arte. Budapest, 649-658 Rowe, C., 2013. La arquitectura de/ siglo XVI en Italia. Barcelona: Editorial Reverté. Vagnetti. L., 1974. L'architecto nella storia di occidente. Florencia: Editorial Teorema, 267. 